\title{
Interleukin-18 and fibroblast growth factor 2 in combination is a useful diagnostic biomarker to distinguish adult-onset Still's disease from sepsis
}

Tomohiro Koga ${ }^{1,2^{*}}$ (D), Remi Sumiyoshi ${ }^{1,3}$, Kaori Furukawa ${ }^{1}$, Shuntaro Sato ${ }^{3}$, Kiyoshi Migita ${ }^{4}$, Toshimasa Shimizu ${ }^{1,3}$, Masataka Umeda', Yushiro Endo ${ }^{1}$, Shoichi Fukui ${ }^{1,5}$, Shin-ya Kawashiri, ${ }^{1,5}$, Naoki Iwamoto ${ }^{1}$, Kunihiro Ichinose ${ }^{1}$, Mami Tamai ${ }^{1}$, Hideki Nakamura ${ }^{1}$, Tomoki Origuchi ${ }^{1}$, Fumiaki Nonaka ${ }^{6}$, Akihiro Yachie ${ }^{7}$, Hideaki Kondo ${ }^{5}$, Takahiro Maeda ${ }^{5,8}$ and Atsushi Kawakami ${ }^{1}$

\begin{abstract}
Objective: To identify potential biomarkers to distinguish adult-onset Still's disease (AOSD) from sepsis.

Method: We recruited 70 patients diagnosed with AOSD according to the Yamaguchi criteria, 22 patients with sepsis, and 118 age-matched controls. Serum levels of 40 cytokines were analyzed using multi-suspension cytokine array. We performed a cluster analysis of each cytokine in the AOSD and sepsis groups in order to identify specific molecular networks. Further, multivariate classification (random forest analysis) and logistic regression analysis were used to rank the cytokines by their importance and determine specific biomarkers for distinguishing AOSD from sepsis.

Results: Seventeen of the 40 cytokines were found to be suitable for further analyses. The serum levels of eleven were significantly higher in patients with AOSD than healthy controls. Levels of serum fibroblast growth factor 2 (FGF-2), vascular endothelial growth factor (VEGF), granulocyte colony-stimulating factor (G-CSF), and interleukin (IL)-18 were significantly elevated in patients with AOSD compared with those with sepsis, and cytokine clustering patterns differed between these two groups. Multivariate classification followed by logistic regression analysis revealed that measurement of both FGF-2 and IL-18 could distinguish AOSD from sepsis with high accuracy (cutoff value for FGF-2 =36 pg/mL; IL-18 = 543 pg/mL, sensitivity 100\%, specificity $72.2 \%$, accuracy $93.8 \%$ ).

Conclusion: Determination of FGF-2 and IL-18 levels in combination may represent a biomarker for the differential diagnosis
\end{abstract} of AOSD from sepsis, based on the measurement of multiple cytokines.

Keywords: Adult-onset Still's disease, Sepsis, IL-18, FGF-2, Cytokine profile

\footnotetext{
* Correspondence: tkoga@nagasaki-u.ac.jp

'Department of Immunology and Rheumatology, Division of Advanced

Preventive Medical Sciences, Nagasaki University Graduate School of

Biomedical Sciences, Nagasaki, Japan

${ }^{2}$ Center for Bioinformatics and Molecular Medicine, Nagasaki University

Graduate School of Biomedical Sciences, 1-12-4 Sakamoto, Nagasaki

852-8523, Japan

Full list of author information is available at the end of the article
}

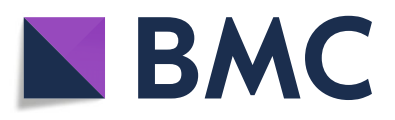

(- The Author(s). 2020 Open Access This article is licensed under a Creative Commons Attribution 4.0 International License, which permits use, sharing, adaptation, distribution and reproduction in any medium or format, as long as you give appropriate credit to the original author(s) and the source, provide a link to the Creative Commons licence, and indicate if changes were made. The images or other third party material in this article are included in the article's Creative Commons licence, unless indicated otherwise in a credit line to the material. If material is not included in the article's Creative Commons licence and your intended use is not permitted by statutory regulation or exceeds the permitted use, you will need to obtain permission directly from the copyright holder. To view a copy of this licence, visit http://creativecommons.org/licenses/by/4.0/. The Creative Commons Public Domain Dedication waiver (http://creativecommons.org/publicdomain/zero/1.0/) applies to the data made available in this article, unless otherwise stated in a credit line to the data. 


\section{Introduction}

Adult-onset Still's disease (AOSD) is a rare autoinflammatory disease characterized by remittent fever, arthritis, a salmon-pink-colored rash in the febrile stage, leukocytosis, lymphadenopathy, and elevated serum ferritin levels [1]. Although the underlying causes of AOSD are currently unknown at present, autoantibodies are not usually detected, and most of AOSD symptoms can be attributed to hypercytokinemia due to abnormal activation of inflammatory cells related to the innate immune system, including neutrophils, monocytes, and macrophages [2].

There are no disease-specific serological markers for the diagnosis of AOSD, and it is considered representative of diseases causing fever of unknown origin (FUO). Thus, early diagnosis is usually difficult, and delayed diagnosis is associated with poor prognosis. Furthermore, severe cases can worsen because of complication with macrophage activation syndrome (MAS) or disseminated intravascular coagulation (DIC) [3]. Early intervention is therefore necessary after appropriate differentiation, in order to improve patient outcomes.

Sepsis is a clinical syndrome that is caused by a dysregulated host response to infection. The release of excess cytokines by activated innate immune cells, particularly macrophages, plays an important role in the pathogenesis of sepsis $[4,5]$. In severe sepsis, such as in AOSD, MAS and DIC are often associated with fatal outcomes owing to multiple organ failure [4]. Thus, some overlap in pathology between sepsis and AOSD may make differentiation clinically difficult, especially in severe cases. A previous report demonstrated that the causative organism is not identified in approximately half of the cases of sepsis [6].

Serum levels of interleukin (IL)-1 $\beta$, IL-6, and IL-18 have been reported to have utility as serum biomarkers for diagnosis and disease evaluation of AOSD [7-10]. However, these cytokines are elevated in a several other inflammatory diseases, including severe infection, and the clinical manifestations of AOSD are also similar to those observed in severe infection. Notably, differentiation of AOSD from sepsis complicated with DIC or presented hyperferritinemia is a major clinical complication. To address this issue, the present study aimed to identify specific biomarkers to distinguish AOSD from sepsis using multivariate analysis combined with the random forest method based on comprehensive analyses of serum cytokines and chemokines.

\section{Methods}

\section{Patients and controls}

This study was registered with the University Hospital Medical Information Network Clinical Trials Registry [http://www.umin.ac.jp/ctr/] as UMIN000030922. We prospectively recruited consecutive patients with AOSD who were treated at Nagasaki University, Shinshu University, Kanazawa University, and Sasebo City Medical Center between April 2014 and October 2018. The diagnosis of AOSD was based on the Yamaguchi criteria [11]. The diagnosis of MAS was confirmed by the findings of hemophagocytosis in the bone marrow aspiration. We also recruited patients with sepsis who were admitted to the rheumatology department of Nagasaki University Hospital between April 2016 and October 2018 and required differential diagnoses from FUO. These patients did not have any other underlying rheumatological conditions. Sepsis was defined according to the Sepsis-3 criteria: increase in Sequential Organ Failure Assessment score by $\geq 2$ at day 1 and suspicion of infection [12]. All participants underwent clinical assessment and provided blood samples for analysis at the time of admission. The control group was recruited from staff at Nagasaki University and residents of the town of Saza in Nagasaki prefecture who underwent specific health checkups in 2016. Inclusion criteria for the control group were no past or present medical histories of inflammatory disease.

All patients provided written informed consent for participation, and the study and all its protocols were approved by the Institutional Review Board of Nagasaki University and related centers (approval no. 180115124). Studies involving the residents of Saza were approved by the Ethics Committee for Human Use of Nagasaki University (approval no. 14051404). Written informed consent was obtained from the residents of Saza who underwent specific health checkups.

\section{Multiplex cytokine and chemokine bead assays}

Serum samples were centrifuged at $3000 \times g$ for $5 \mathrm{~min}$, and the supernatants were collected and stored at $80{ }^{\circ} \mathrm{C}$ for a maximum of 90 days prior to analysis. A blinded multiplex cytokine bead assay was performed in parallel using the Bio-plex MAGPIX ${ }^{\mathrm{TM}}$ Human Cytokine assay (Bio-Rad, Hercules, CA, USA) and MILLIPLEX MAP Human Cytokine/Chemokine Magnetic Bead Panel 1-Premixed 38 Plex (Millipore, Billerica, MA, USA) kits, according to the manufacturers' instructions. Cytokines that were frequently found to be at nondetectable levels were excluded from analysis.

\section{Statistical analysis}

Baseline demographic characteristics and cytokine/chemokine levels of the study population were compared using the Kruskal-Wallis test followed by Dunn's multiple comparisons test. Correlations between pairs of serum markers were calculated using Spearman's rank correlation test. To rank the cytokine levels, we performed the multivariate classification algorithm of 
random forest analysis (RFA) using the $\mathrm{R}$ package RandomForest (http://cran.r-project.org/web/packages/randomForest/) version 4.6.12 software, as previously described [13]. We subsequently selected a classifier consisting of a combination of cytokine markers that yielded the best classification performance to predict AOSD by multiple logistic regression analysis. We then calculated the sensitivity, specificity, accuracy, receiver operator characteristic (ROC) curve, area under the curve (AUC), and Akaike's information criterion (AIC). Statistical analyses were performed using $\mathrm{R}$ software (version 3.2.3) and JMP pro (version 13.0) software (SAS Institute, Cary, $\mathrm{NC}, \mathrm{USA}$ ). All reported $p$ values are twosided, and a $p$ value of $<0.05$ was considered statistically significant. Bonferroni's correction for multiple-cytokine testing $(n=17)$ was applied, and $p<0.00284$ was considered significant.

\section{Results}

\section{Study population}

The study population comprised 70 patients with AOSD, 22 with sepsis, and 118 age- and sex-matched healthy controls. Table 1 presents the demographic, clinical, and laboratory characteristics of patients with AOSD and sepsis. All patients had a treatment course of $<1$ month, and none of the patients had undergone treatment with glucocorticoids or other immunosuppressive agents. The median ages at diagnosis were 48 years and 59 years in the AOSD and the sepsis groups, respectively.

\section{Cytokine profiles of patients with AOSD, sepsis, and healthy controls}

After exclusion of cytokines that were frequently nondetectable, we were able to analyze 17 cytokines: EOTAXIN (CCL11), fibroblast growth factor 2 (FGF-2), basic granulocyte colony-stimulating factor (G-CSF), granulocyte macrophage colony-stimulating factor (GMCSF), CXCL1 (growth-regulated protein alpha precursor [GRO]), interferon- $\gamma$ (IFN- $\gamma$ ), IL-17, IL-18, IL-6, IL-8, CXCL10 (interferon gamma-inducible protein 10 [IP10]), CCL2 (monocyte chemoattractant protein-1 [MCP1]/MCAF), CCL22 (human macrophage-derived chemokine $[\mathrm{MDC}]$ ), CCL3 (macrophage inflammatory protein1a (MIP-1a]), CCL4 (macrophage inflammatory protein$1 \mathrm{~b}$ [MIP-1b]), TNF- $\alpha$, and vascular endothelial growth factor (VEGF).

Serum levels of four cytokines were significantly elevated in patients with AOSD compared with those with sepsis (median FGF-2, $48.7 \mathrm{pg} / \mathrm{mL}$ vs. 25.7, $p<0.0001$; median GM-CSF, $13.1 \mathrm{pg} / \mathrm{mL}$ vs. $1.8 \mathrm{pg} / \mathrm{mL}, p<0.0001$; median IL-18, $12,070 \mathrm{pg} / \mathrm{mL}$ vs. $104.7 \mathrm{pg} / \mathrm{mL}, p<0.0001$; and median VEGF, $333.3 \mathrm{pg} / \mathrm{mL}$ vs. $28.6 \mathrm{pg} / \mathrm{mL}, p<$ 0.0001) (Fig. 1). Eleven cytokines (FGF-2, G-CSF, GMCSF, CXCL1, IFN- $\gamma$, IL-17, IL-6, IL-8, CXCL10, TNF- $\alpha$, and VEGF) were significantly increased in the AOSD group compared with the control group (Table 2).

\section{Comparison of activated cytokine networks between patients with AOSD and patients with sepsis}

To compare activated cytokine networks between patients with AOSD and those with sepsis, we further examined the correlations between serum levels of activated individual cytokines in patients with AOSD and in patients with sepsis. We found significant correlations between IL-17 and IL-8 $(r=0.819, p<0.0001)$, TNF- $\alpha$ and IFN- $\gamma(r=$ $0.640, p<0.0001)$, VEGF and IL-17 $(r=0.585, p<0.0001)$, FGF-2 and GM-CSF $(r=0.5843, p<0.0001)$, and VEGF and IL-8 $(r=0.545, p<0.0001)$ in patients with AOSD.

In the sepsis group, significant correlations were found between IFN- $\gamma$ and TNF- $\alpha(r=0.832, p<0.0001)$, GM-CSF and FGF-2 $(r=0.794, p<0.0001)$, G-CSF and IL-6 $(r=0.626$, $p=0.0054)$, G-CSF and IL-18 $(r=0.572, p=0.013)$, IL-8 and

Table 1 The demographic, clinical, and laboratory characteristics of patients with AOSD and sepsis

\begin{tabular}{lllcr}
\hline Characteristic & AOSD patients $(n=70)$ & Sepsis patients $(n=22)$ & Healthy controls $(n=118)$ & $p$ value (AOSD vs. sepsis) \\
\hline Age at diagnosis, years & $48(33-65)$ & $59(44-71)$ & $56(47-65)$ & 0.12 \\
Female, $n$ (\%) & $44(71)$ & $12(54)$ & & 0.17 \\
Ferritin at diagnosis, $\mathrm{ng} / \mathrm{mL}$ & $4019(1184-11,870)$ & & \\
CRP at diagnosis, $\mathrm{mg} / \mathrm{L}$ & $87(48-135)$ & & \\
ESR at diagnosis, $\mathrm{mm} / \mathrm{h}$ & $48(34-73)$ & $15,300(13,625-18,500)$ & 0.056 \\
WBC at diagnosis, $/ \mu \mathrm{L}$ & $12,290(6690-17,455)$ & & 0.12 \\
AST at diagnosis, U/L & $50(31-93)$ & & \\
ALT at diagnosis, U/L & $36(17-112)$ & $3(2.8-4.3)$ & \\
SOFA score & $3(4)$ & $3(17)$ & 0.26 \\
DIC, $n$ (\%) & $5(7)$ & $0(0)$ & \\
MAS, $n(\%)$ & & &
\end{tabular}

CRP C-reactive protein, ESR erythrocyte sedimentation rate, WBC white blood cell, AST aspartate aminotransferase, ALT alanine animotransferase, SOFA Sequential Organ Failure Assessment, DIC disseminated intravascular coagulation, MAS macrophage activation syndrome 


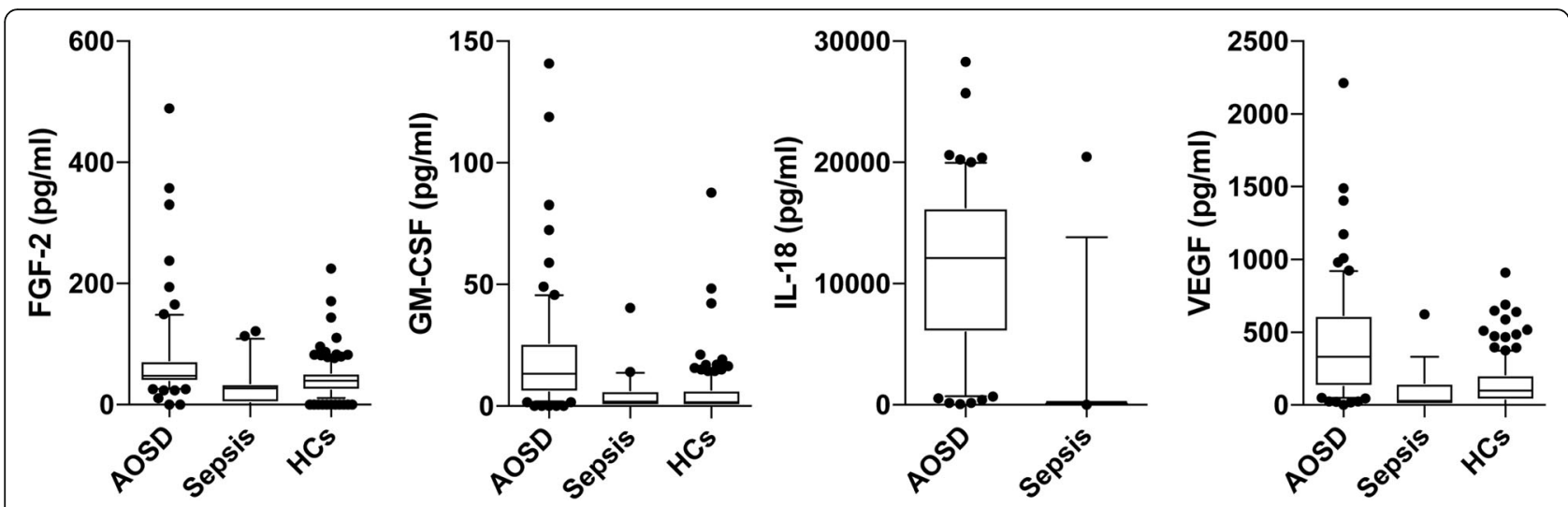

Fig. 1 A multiplex cytokine bead assay of FGF-2, GM-CSF, IL-18, and VEGF in the serum of patients with AOSD and sepsis. Data are presented as box and whisker plots [median, interquartile range, and range (10-90 percentiles)]

TNF- $\alpha(r=0.5454, p=0.0192)$, IP-10 and TNF- $\alpha(r=0.537$, $p=0.022)$, and IL-8 and IFN- $\gamma(r=0.530, p=0.024)$. Hierarchical clustering with heatmaps based on the Pearson correlation coefficients is shown in Fig. 2a (for the AOSD group) and Fig. $2 \mathrm{~b}$ (for the sepsis group).

These results suggest that the cytokine networks in patients with AOSD differ from those in patients with sepsis. Thus, in the AOSD group, FGF-2, GM-CSF, IL-17, IL-18, and VEGF form interrelated networks, whereas in the sepsis group, IFN- $\gamma$, TNF- $\alpha$, IL-8, IL10 , and IL-18 form interrelated networks.

\section{Identification of combinational biomarkers for the differential diagnosis of AOSD from sepsis by RFA and logistic regression analysis}

The results of ranking of cytokines by importance according to RFA are illustrated in Fig. 2c. IL-18 and then

Table 2 Cytokine profile of patients with AOSD, those with sepsis, and the healthy controls

\begin{tabular}{|c|c|c|c|c|c|c|}
\hline \multirow[t]{2}{*}{ Cytokine } & \multirow[t]{2}{*}{$\mathrm{HCS}(n=118)$} & \multirow[t]{2}{*}{$\operatorname{AOSD}(n=70)$} & \multirow[t]{2}{*}{ Sepsis $(n=18)$} & \multicolumn{3}{|l|}{$p$ value } \\
\hline & & & & AOSD vs. sepsis & Sepsis vs. HCs & AOSD vs. HCs \\
\hline EOTAXIN (CCL11) & $137.5(95.1-186.4)$ & $104.0(66.0-134.0)$ & $123.4(70.5-172.5)$ & 0.41 & 0.54 & 0.01 \\
\hline FGF-2 & $37.7(21.2-58.1)$ & $48.7(41.1-74.4)$ & $25.7(11.1-34.1)$ & $<0.0001$ & 0.0057 & $<0.0001$ \\
\hline G-CSF & $8.2(0.1-22.5)$ & $100.9(50.5-173.6)$ & $124.1(45.9-255.2)$ & 0.69 & $<0.0001$ & $<0.0001$ \\
\hline GM-CSF & $1.5(0.1-6.7)$ & $13.1(5.7-26.8)$ & $1.8(0.1-3.2)$ & $<0.0001$ & 0.41 & $<0.0001$ \\
\hline GRO (CXCL1) & $978.1(775.5-1237)$ & $1488(1034-2383)$ & $697.2(432.1-1449)$ & 0.027 & 0.25 & $<0.0001$ \\
\hline $\mathrm{IFN}-\gamma$ & $4.7(1.5-11.3)$ & $22.7(7.5-60.2)$ & $12.5(3.0-21.1)$ & 0.052 & 0.13 & $<0.0001$ \\
\hline $\mathbb{I L}-17$ & $1.9(0.3-6.2)$ & $5.3(0.9-18.5)$ & $0.13(0.1-7.4)$ & 0.019 & 0.29 & 0.0002 \\
\hline IL-18 & N/A & $12,070(6027-16,451)$ & $104.7(75.1-382.1)$ & $<0.0001$ & N/A & N/A \\
\hline IL-6 & $0.1(0-0.1)$ & $31.7(12.3-69.7)$ & $28.1(0.7-100.5)$ & 0.55 & $<0.0001$ & $<0.0001$ \\
\hline IL-8 & $63.0(28.3-108.3)$ & $30.5(15.6-50.9)$ & $49.8(35.7-56.7)$ & 0.96 & 0.44 & $<0.0001$ \\
\hline IP-10 (CXCL10) & $277.9(221.0-351.8)$ & $1406(661.8-3806)$ & $2082(542.3-4212)$ & 0.81 & $<0.0001$ & $<0.0001$ \\
\hline MCP-1 (CCL2) & $655.9(530.5-838.7)$ & $790.8(475-1675)$ & $1510(811-3281)$ & 0.13 & 0.027 & 0.011 \\
\hline MDC (CCL2) & 848.95 (703.0-1008) & $668(338.5-1013)$ & $352.3(753.7-1013)$ & 0.14 & $<0.0001$ & 0.0036 \\
\hline MIP-1a (CCL3) & $14.9(0.1-29.4)$ & $14.2(1.1-20.1)$ & $18.6(3.4-35.3)$ & 0.37 & 0.51 & 0.42 \\
\hline MIP-1b (CCL4) & $59.6(32.3-82.8)$ & $62.1(39.6-86.2)$ & $40.3(9.5-59.0)$ & 0.11 & 0.16 & 0.13 \\
\hline TNF-a & $12.6(9.3-16.7)$ & $30.1(18.1-68.2)$ & $26.9(16.7-73.9)$ & 0.71 & $<0.0001$ & $<0.0001$ \\
\hline VEGF & 99.4 (31.9-208.5) & 333.3 (128.0-616.4) & $28.6(0.1-151.2)$ & $<0.0001$ & 0.083 & $<0.0001$ \\
\hline
\end{tabular}

Values are the median (interquartile range) $\mathrm{pg} / \mathrm{mL}$. $p$ values were established using the Kruskal-Wallis test followed by a Dunn's multiple comparisons test. FGF fibroblast growth factor, GM-CSF granulocyte macrophage colony-stimulating factor, GRO growth-regulated protein alpha precursor, G-CSF granulocyte colonystimulating factor, IL interleukin, MCP-1 monocyte chemoattractant protein-1, MDC human macrophage-derived chemokine, TNF-a tumor necrosis factor-alpha, VEGF vascular endothelial growth factor 
A

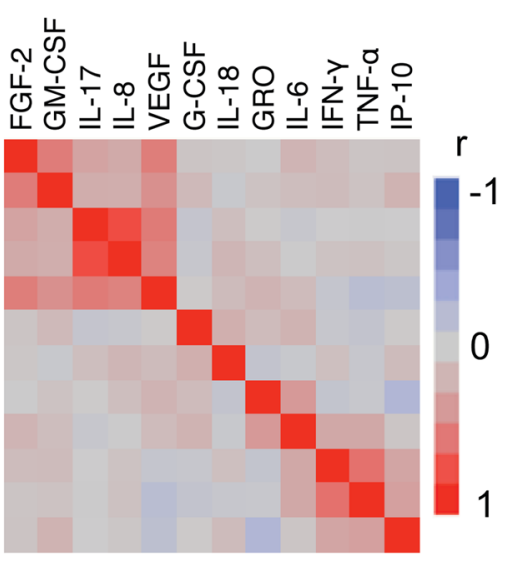

B

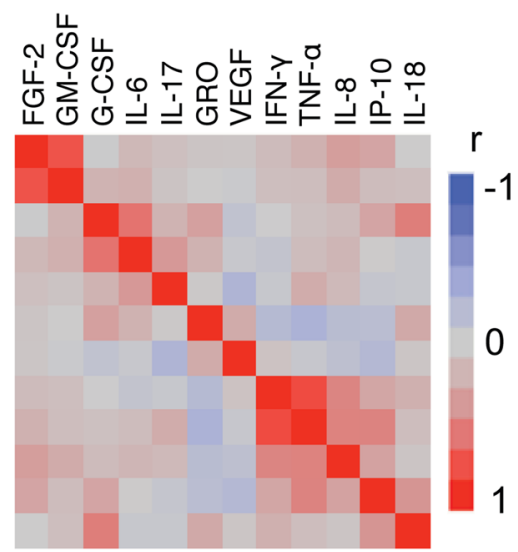

C

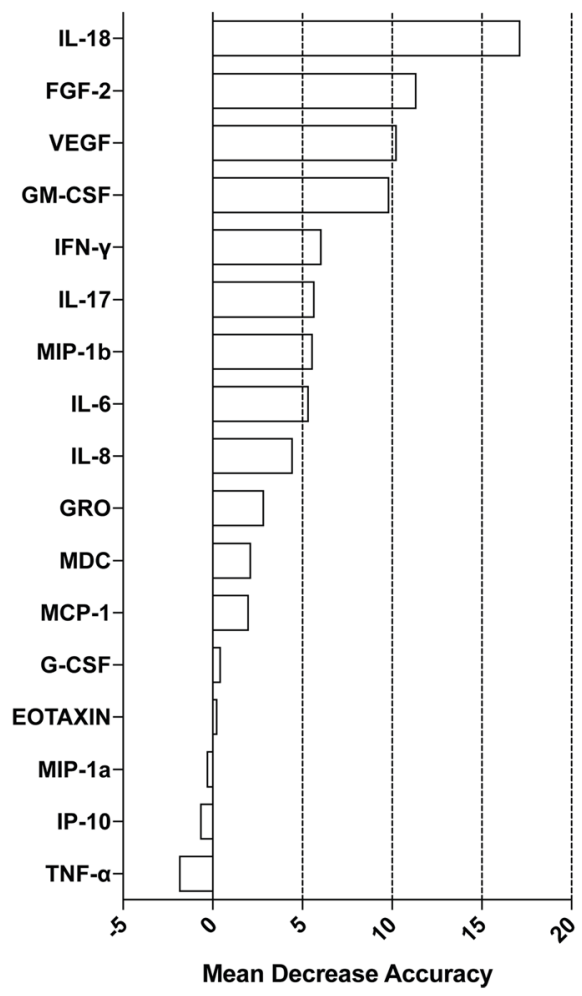

Fig. 2 Cytokine networks in the patients with AOSD and sepsis. Hierarchical clustering with a Pearson correlation heatmap of serum cytokine levels among patients with $\mathbf{a}$ AOSD and $\mathbf{b}$ sepsis. c Cytokines ranked by their relative importance for discriminating AOSD from sepsis. The horizontal axis represents the average decrease in classification accuracy

FGF-2 were extracted as the most important cytokines for distinguishing AOSD from sepsis (mean decrease accuracy 17.2 and 11.4, respectively). The results of multiple logistic regression analysis and ROC curves for sensitivity, specificity, accuracy, AUC, and AIC are shown in Table 3 . The best combination of cytokines to distinguish AOSD from sepsis was found to be IL-18 (> $543 \mathrm{pg} / \mathrm{mL})$ and FGF-2 (> $36 \mathrm{pg} / \mathrm{mL})$, with high accuracy observed (sensitivity $100 \%$, specificity $72.2 \%$, and accuracy $93.8 \%$; Table 3 ).

\section{Discussion}

Infections, malignancies, and systemic rheumatic diseases including AOSD generally account for most cases of FUO. An important aspect in the management of FUO is accurate diagnosis of AOSD, which requires

Table 3 ROC curve in each subset determined by multiple logistic regression analysis

\begin{tabular}{|c|c|c|c|c|c|c|}
\hline Variables (AOSD vs. sepsis) & Sensitivity & Specificity & Accuracy & AUC & AlC & Cutoff value $(\mathrm{pg} / \mathrm{mL})$ \\
\hline $\mathrm{IL}-18$ & 93.7 & 83.3 & 91.4 & 0.884 & 65.67 & 543 \\
\hline GM-CSF & 85.3 & 83.3 & 84.9 & 0.88 & 61.33 & 4.4 \\
\hline FGF-2 & 82.4 & 88.9 & 83.7 & 0.864 & 62.5 & 36 \\
\hline VEGF & 66.2 & 88.9 & 70.9 & 0.85 & 70.55 & 221 \\
\hline $\mathrm{IL}-18>543 \mathrm{pg} / \mathrm{mL}+\mathrm{FGF}-2>36 \mathrm{pg} / \mathrm{mL}$ & 100 & 72.2 & 93.8 & 0.966 & 29.7 & \\
\hline $\mathrm{IL}-18>543 \mathrm{pg} / \mathrm{mL}+\mathrm{GM}-\mathrm{CSF}>4.4 \mathrm{pg} / \mathrm{mL}$ & 92.1 & 83.3 & 90.1 & 0.944 & 37.1 & \\
\hline $\mathrm{FGF}-2>36 \mathrm{pg} / \mathrm{mL}+\mathrm{GM}-\mathrm{CSF}>4.4 \mathrm{pg} / \mathrm{mL}$ & 89.7 & 83.3 & 88.4 & 0.877 & 59.32 & \\
\hline
\end{tabular}

Data in boldface indicates the minimum number of cytokines among the subsets. AIC Akaike's information criterion, AUC area under the curve, FGF-2 fibroblast growth factor 2, GM-CSF granulocyte macrophage colony-stimulating factor 
exclusion of infection. It is often difficult to distinguish AOSD from infection because clinical manifestations of AOSD may mimic those of some infections such as sepsis. The similarities between these two diseases include laboratory findings such as marked neutrophilia, increased liver enzymes, or hyperferritinemia. To date, there have been few studies focusing on biomarkers to distinguish the two conditions, and-to the best of our knowledge-there have been no comprehensive analyses involving large numbers of patients with untreated AOSD. The present evaluation of cytokine networks using a multi-suspension cytokine array system resulted in the identification of possible diagnostic biomarkers to distinguish AOSD from sepsis with high accuracy.

Of the biomarkers that were identified, IL-18 is a proinflammatory cytokine belonging to the IL- 1 family that induces the production of IFN- $\gamma$ via inflammasome signals triggered by pathogen-associated or damageassociated molecular patterns (PAMPs or DAMPs) [14]. IL-18 is produced by a variety of cells, including monocytes, macrophages, and dendritic cells [15], and is thought to play a major role in the development of AOSD. In addition to the significant increase in serum levels of IL-18 in patients with AOSD and its correlation with disease activity $[9,16]$, skin and synovial biopsies from patients with active AOSD showed higher expression levels of IL-18 mRNA compared with controls [9]. In addition, IL-18 has been shown to be overexpressed in other sites, such as lymph nodes and liver, in patients with AOSD [17, 18]. AOSD has been considered an autoinflammatory disease caused by inflammasome activation because of the utility of IL-1 inhibitors [19] and the lack of significant increase of autoantibodies levels [20]. In the pathogenesis of AOSD, IL-18, which is produced by NLPR3 inflammasome activation [21], induces the production of IFN- $\gamma$ by activating T cells with IL-18 receptors. IL-18 also plays an important role in the development of MAS in patients with AOSD [22].

Accordingly, in contrast with inflammatory cytokines such as IL-1 $\beta$, IL- 6 , TNF- $\alpha$, and IFN- $\gamma$, elevation of IL18 has levels been reported to be characteristic of AOSD [7] and, thus, potentially useful for differentiating AOSD from sepsis [23]. However, the production of IL-18 is increased by lipopolysaccharide-stimulated NLRP3 inflammasome activation [24], indicating that IL-18 levels are increased in patients with sepsis [25]. In line with this observation, serum IL-18 levels of patients with sepsis were elevated to the same levels as in patients with AOSD in some cases in the present study.

Previous studies showed that serum IL-18 levels in patients with AOSD varied widely, from $788 \mathrm{pg} / \mathrm{mL}$ (mean value) to $16,327 \mathrm{pg} / \mathrm{mL}$ (median value) $[7,9,16,17,23$, 26-28]. The median IL-18 level in this study was 12,070 $\mathrm{pg} / \mathrm{mL}$, which was higher than that reported in some studies $[7,9,16,17,23,26,27]$. We speculate that this may be due to differences in the detection range of ELISA and bead array tests as well as differences in patient characteristics. IL-18 levels tended to be higher, especially in patients with AOSD with high disease activity $[9,16]$, suggesting that high disease activity in the patient population may have influenced these variations.

In contrast to our findings, Rau et al. reported no significant differences in serum IL-18 levels between patients with sepsis and those with AOSD [29]. Although this result differs from our results, a major limitation of that study was the small number of patients in the study cohort (18 patients with AOSD and 14 patients with sepsis). In addition, as the authors emphasize, the wide range of IL-18 levels observed in all patient groups may have biased the statistical analysis. In another study comparing patients with sepsis and AOSD, serum IL-18 levels were higher in patients with AOSD than in those with sepsis [23], which is consistent with our results.

The biological activity of IL-18 is tightly regulated by IL-18 binding protein (IL-18 BP), a natural inhibitor that binds IL-18 with high affinity [30]. Although serum levels of free IL-18 have been shown to be elevated in AOSD and correlate with clinical and biological markers of disease activity [7,31], we have not been able to accurately assess IL-18 activity because the assay used in this study cannot distinguish free IL-18 that is not bound to IL-18 BP (active) and IL-18 complexed with IL-18 BP (inactive). This is considered to be a limitation of this study.

Although, consistent with previous reports [32], we did not observe serum FGF-2 levels to be elevated in patients with sepsis, no studies have measured serum FGF-2 levels in patients with AOSD. This is therefore the first study to suggest FGF-2 as a new biomarker for AOSD. This cytokine is produced by a number of cell types, including fibroblasts, endothelial cells, and macrophages; it represents the most potent inducer of angiogenesis and is released following tissue injuries and during inflammation [33]. While FGF-2 is induced by IL-1 $\beta$ in human osteoblasts and fibroblasts [34], the cytokine is induced by IL-1 $\beta$ in endothelial cells [35]. Patients with AOSD exhibit synovial hyperplasia and synovitis similar to rheumatoid arthritis (RA) due to the activation of synovial fibroblasts. Elevated serum levels of soluble intercellular adhesion molecule-1 (sICAM-1) have also been reported in the context of AOSD, suggesting that vascular endothelial cells may be activated. Collectively, our findings lead us to speculate that these cells may be the primary source of FGF-2 in patients with AOSD. The most common pathway employed by FGF-2 is the p38 mitogen-activated protein kinase (MAPK) pathway [36], which has been suggested to exacerbate arthritis and bone destruction in RA. Therefore, it is proposed that high levels of FGF-2 may be involved in promoting p38 
MAPK-mediated arthritis and inflammatory responses in patients with AOSD.

Our study has several limitations that should be acknowledged. The cross-sectional design and use of serum samples from patients with untreated AOSD mean that continuous serum samples were not used in this study. Although it has been suggested that IL-18 is useful as a biomarker to reflect the therapeutic response and disease activity of AOSD [28, 31, 37] and a phase II clinical trial showed that IL-18 inhibition by administration of recombinant human IL-18BP is effective in the treatment of AOSD [38], further longitudinal studies are needed to verify whether the cytokines identified in this study are associated with therapeutic responses. In addition, the cytokine profiles presented here for patients with sepsis may differ from those of normal populations of patients with sepsis because we included patients admitted for evaluation of FUO who were subsequently diagnosed with sepsis. Therefore, studies involving more patients with sepsis should be conducted in the future. Finally, although the efficacy of an IL-1 inhibitor in patients with severe AOSD has been shown [19], the serum IL-1 $\beta$ level is not high enough for the assay that we used to detect significant differences.

\section{Conclusions}

In conclusion, our study demonstrates that the measurement of FGF-2 and IL-18 levels in combination represents an effective biomarker for the differential diagnosis of AOSD from sepsis. Although differential diagnosis from rheumatic diseases and infectious conditions poses considerable challenges in clinical practice, the findings of this study may help to enhance the diagnostic performance of AOSD in daily practice and drive the discovery of further biomarkers.

\footnotetext{
Abbreviations

AIC: Akaike's information criterion; AOSD: Adult-onset Still's disease; AUC: Area under the curve; DIC: Disseminated intravascular coagulation; FGF2: Fibroblast growth factor 2; FUO: Fever of unknown origin; GCSF: Granulocyte colony-stimulating factor; GM-CSF: Granulocyte macrophage colony-stimulating factor; IL: Interleukin; MAS: Macrophage activation syndrome; MIP: Macrophage inflammatory protein; RA: Rheumatoid arthritis; RFA: Random forest analysis; ROC: Receiver operator characteristic; VEGF: Vascular endothelial growth factor
}

\section{Acknowledgements}

The authors wish to thank the patients and medical staff for their contributions to the study.

\section{Authors' contributions}

Dr. Koga had full access to all of the data in the study and takes responsibility for the integrity of the data and the accuracy of the data analysis. Study design: T. Koga, A. Kawakami. Acquisition of data: T. Koga, R. Sumiyoshi, K. Furukawa, K. Migita, T. Shimizu, M. Umeda, Y. Endo, S. Fukui, SY. Kawashiri, N. Iwamoto, K. Ichinose, M. Tamai, H. Nakamura, T. Origuchi, F. Nonaka, A. Yachie, H. Kondo, T. Maeda, A. Kawakami. Analysis and interpretation of data: T. Koga. Manuscript preparation: T. Koga. Statistical analysis: T. Koga, S. Sato. The authors read and approved the final manuscript.

\section{Funding}

This work was supported by the Japan Agency for Medical Research and Development (grant no. 15657398 to AK) and NOVARTIS Foundation (Japan) for the Promotion of Science 2018 (to TK).

\section{Availability of data and materials}

The datasets used and/or analyzed during the present study are available from the corresponding author on reasonable request.

\section{Ethics approval and consent to participate}

The study was approved by the medical ethics committee of Nagasaki University and related centers. All patients gave signed informed consent.

Consent for publication

All authors consented to the publication of this manuscript.

\section{Competing interests}

The authors declare that there are no competing interests.

\section{Author details}

'Department of Immunology and Rheumatology, Division of Advanced Preventive Medical Sciences, Nagasaki University Graduate School of Biomedical Sciences, Nagasaki, Japan. ${ }^{2}$ Center for Bioinformatics and Molecular Medicine, Nagasaki University Graduate School of Biomedical Sciences, 1-12-4 Sakamoto, Nagasaki 852-8523, Japan. ${ }^{3}$ Clinical Research Center, Nagasaki University Hospital, Nagasaki, Japan. ${ }^{4}$ Department of Rheumatology, Fukushima Medical University School of Medicine, Fukushima, Japan. ${ }^{5}$ Department of Community Medicine, Nagasaki University Graduate School of Biomedical Sciences, Nagasaki, Japan. ${ }^{6}$ Department of Internal Medicine, Sasebo City General Hospital, Sasebo, Japan. ${ }^{7}$ Department of Pediatrics, School of Medicine, Institute of Medical, Pharmaceutical and Health Sciences, Kanazawa University, Kanazawa, Japan. ${ }^{8}$ Department of General Medicine, Nagasaki University Hospital, Nagasaki University Graduate School of Biomedical Sciences, Nagasaki, Japan.

Received: 20 February 2020 Accepted: 27 April 2020

Published online: 07 May 2020

\section{References}

1. Gerfaud-Valentin M, Jamilloux Y, Iwaz J, Seve P. Adult-onset Still's disease. Autoimmun Rev. 2014;13(7):708-22

2. Ohta A, Yamaguchi M, Tsunematsu T, Kasukawa R, Mizushima H, Kashiwagi H, Kashiwazaki S, Tanimoto K, Matsumoto Y, Akizuki M, et al. Adult Still's disease: a multicenter survey of Japanese patients. J Rheumatol. 1990;17(8): 1058-63.

3. Arlet JB, Le TH, Marinho A, Amoura Z, Wechsler B, Papo T, Piette JC. Reactive haemophagocytic syndrome in adult-onset Still's disease: a report of six patients and a review of the literature. Ann Rheum Dis. 2006;65(12): 1596-601.

4. Karakike E, Giamarellos-Bourboulis EJ. Macrophage activation-like syndrome: a distinct entity leading to early death in Sepsis. Front Immunol. 2019;10:55.

5. Cinel I, Dellinger RP. Advances in pathogenesis and management of sepsis. Curr Opin Infect Dis. 2007;20(4):345-52.

6. Gupta S, Sakhuja A, Kumar G, McGrath E, Nanchal RS, Kashani KB. Culturenegative severe sepsis: nationwide trends and outcomes. Chest. 2016;150(6): 1251-9.

7. Girard C, Rech J, Brown M, Allali D, Roux-Lombard P, Spertini F, Schiffrin EJ, Schett G, Manger B, Bas S, et al. Elevated serum levels of free interleukin-18 in adult-onset Still's disease. Rheumatology (Oxford). 2016;55(12):2237-47.

8. Feist E, Mitrovic S, Fautrel B. Mechanisms, biomarkers and targets for adultonset Still's disease. Nat Rev Rheumatol. 2018;14(10):603-18.

9. Chen DY, Lan JL, Lin FJ, Hsieh TY. Proinflammatory cytokine profiles in sera and pathological tissues of patients with active untreated adult onset Still's disease. J Rheumatol. 2004;31(11):2189-98.

10. Choi JH, Suh CH, Lee YM, Suh YJ, Lee SK, Kim SS, Nahm DH, Park HS. Serum cytokine profiles in patients with adult onset Still's disease. J Rheumatol. 2003:30(11):2422-7.

11. Yamaguchi M, Ohta A, Tsunematsu T, Kasukawa R, Mizushima Y, Kashiwagi H, Kashiwazaki S, Tanimoto K, Matsumoto Y, Ota T, et al. Preliminary criteria for classification of adult Still's disease. J Rheumatol. 1992;19(3):424-30. 
12. Singer M, Deutschman CS, Seymour CW, Shankar-Hari M, Annane D, Bauer M, Bellomo R, Bernard GR, Chiche JD, Coopersmith CM, et al. The third international consensus definitions for sepsis and septic shock (Sepsis-3). JAMA. 2016;315(8):801-10.

13. Koga T, Migita K, Sato S, Umeda M, Nonaka F, Kawashiri SY, Iwamoto N, Ichinose K, Tamai M, Nakamura $\mathrm{H}$, et al. Multiple serum cytokine profiling to identify combinational diagnostic biomarkers in attacks of familial Mediterranean fever. Medicine. 2016;95(16):e3449.

14. Jamilloux Y, Gerfaud-Valentin M, Martinon F, Belot A, Henry T, Seve P. Pathogenesis of adult-onset Still's disease: new insights from the juvenile counterpart. Immunol Res. 2015;61(1-2):53-62.

15. Dinarello CA, Novick D, Kim S, Kaplanski G. Interleukin-18 and IL-18 binding protein. Front Immunol. 2013;4:289.

16. Fujii T, Nojima T, Yasuoka H, Satoh S, Nakamura K, Kuwana M, Suwa A, Hirakata M, Mimori T. Cytokine and immunogenetic profiles in Japanese patients with adult Still's disease. Association with chronic articular disease. Rheumatology (Oxford). 2001;40(12):1398-404

17. Conigliaro P, Priori R, Bombardieri M, Alessandri C, Barone F, Pitzalis C, McInnes IB, Valesini G. Lymph node IL-18 expression in adult-onset Still's disease. Ann Rheum Dis. 2009;68(3):442-3.

18. Priori $R$, Barone F, Alessandri C, Colafrancesco S, Mclnnes IB, Pitzalis $C$, Valesini G, Bombardieri M. Markedly increased IL-18 liver expression in adultonset Still's disease-related hepatitis. Rheumatology (Oxford). 2011;50(4): 776-80.

19. Cavalli G, Tomelleri A, De Luca G, Campochiaro C, Dinarello CA, Baldissera E, Dagna L. Efficacy of canakinumab as first-line biologic agent in adult-onset Still's disease. Arthritis Res Ther. 2019;21(1):54.

20. Rossi-Semerano L, Kone-Paut I. Is Still's disease an autoinflammatory syndrome? Int J Inflam. 2012;2012:480373.

21. Hsieh CW, Chen YM, Lin CC, Tang KT, Chen HH, Hung WT, Lai KL, Chen DY. Elevated expression of the NLRP3 inflammasome and its correlation with disease activity in adult-onset Still disease. J Rheumatol. 2017:44(8):1142-50.

22. Ichida H, Kawaguchi Y, Sugiura T, Takagi K, Katsumata Y, Gono T, Ota Y, Kataoka S, Kawasumi H, Yamanaka H. Clinical manifestations of adult-onset Still's disease presenting with erosive arthritis: association with low levels of ferritin and Interleukin-18. Arthritis Care Res (Hoboken). 2014;66(4):642-6.

23. Priori R, Colafrancesco S, Alessandri C, Minniti A, Perricone C, laiani G, Palazzo D, Valesini G. Interleukin 18: a biomarker for differential diagnosis between adult-onset Still's disease and sepsis. J Rheumatol. 2014;41(6): $1118-23$.

24. Jiang L, Zhang L, Kang K, Fei D, Gong R, Cao Y, Pan S, Zhao M, Zhao M. Resveratrol ameliorates LPS-induced acute lung injury via NLRP3 inflammasome modulation. Biomed Pharmacother. 2016;84:130-8.

25. Grobmyer SR, Lin E, Lowry SF, Rivadeneira DE, Potter S, Barie PS, Nathan CF. Elevation of IL-18 in human sepsis. J Clin Immunol. 2000;20(3):212-5.

26. Kawaguchi $Y$, Terajima H, Harigai M, Hara M, Kamatani N. Interleukin-18 as a novel diagnostic marker and indicator of disease severity in adult-onset Still's disease. Arthritis Rheum. 2001;44(7):1716-7.

27. Chen DY, Lan JL, Lin FJ, Hsieh TY. Association of intercellular adhesion molecule-1 with clinical manifestations and interleukin-18 in patients with active, untreated adult-onset Still's disease. Arthritis Rheum. 2005;53(3):320-7.

28. Kudela H, Drynda S, Lux A, Horneff G, Kekow J. Comparative study of Interleukin-18 (IL-18) serum levels in adult onset Still's disease (AOSD) and systemic onset juvenile idiopathic arthritis $(\mathrm{s} / \mathrm{A})$ and its use as a biomarker for diagnosis and evaluation of disease activity. BMC Rheumatol. 2019;3:4.

29. Rau M, Schiller M, Krienke S, Heyder P, Lorenz H, Blank N. Clinical manifestations but not cytokine profiles differentiate adult-onset Still's disease and sepsis. J Rheumatol. 2010;37(11):2369-76.

30. Novick D, Kim SH, Fantuzzi G, Reznikov LL, Dinarello CA, Rubinstein M. Interleukin-18 binding protein: a novel modulator of the Th1 cytokine response. Immunity. 1999;10(1):127-36.

31. Jung KH, Kim JJ, Lee JS, Park W, Kim TH, Jun JB, Yoo DH. Interleukin-18 as an efficient marker for remission and follow-up in patients with inactive adult-onset Still's disease. Scand J Rheumatol. 2014;43(2):162-9.

32. Faiotto VB, Franci D, Enz Hubert RM, de Souza GR, Fiusa MML, Hounkpe BW, Santos TM, Carvalho-Filho MA, De Paula EV. Circulating levels of the angiogenesis mediators endoglin, HB-EGF, BMP-9 and FGF-2 in patients with severe sepsis and septic shock. J Crit Care. 2017:42:162-7.

33. Shute J, Marshall L, Bodey K, Bush A. Growth factors in cystic fibrosis - when more is not enough. Paediatr Respir Rev. 2003:4(2):120-7.
34. Sobue T, Zhang X, Florkiewicz RZ, Hurley MM. Interleukin-1 regulates FGF-2 mRNA and localization of FGF-2 protein in human osteoblasts. Biochem Biophys Res Commun. 2001;286(1):33-40.

35. Lee HT, Lee JG, Na M, Kay EP. FGF-2 induced by interleukin-1 beta through the action of phosphatidylinositol 3-kinase mediates endothelial mesenchymal transformation in corneal endothelial cells. J Biol Chem. 2004; 279(31):32325-32

36. Clark AR, Dean JL. The p38 MAPK pathway in rheumatoid arthritis: a sideways look. Open Rheumatol J. 2012;6:209-19.

37. Colafrancesco S, Priori R, Alessandri C, Perricone C, Pendolino M, Picarelli G, Valesini G. IL-18 serum level in adult onset Still's disease: a marker of disease activity. Int J Inflam. 2012;2012:156890.

38. Gabay C, Fautrel B, Rech J, Spertini F, Feist E, Kotter I, Hachulla E, Morel J, Schaeverbeke T, Hamidou MA, et al. Open-label, multicentre, doseescalating phase $\|$ clinical trial on the safety and efficacy of tadekinig alfa (IL-18BP) in adult-onset Still's disease. Ann Rheum Dis. 2018;77(6):840-7.

\section{Publisher's Note}

Springer Nature remains neutral with regard to jurisdictional claims in published maps and institutional affiliations.
Ready to submit your research? Choose BMC and benefit from:

- fast, convenient online submission

- thorough peer review by experienced researchers in your field

- rapid publication on acceptance

- support for research data, including large and complex data types

- gold Open Access which fosters wider collaboration and increased citations

- maximum visibility for your research: over $100 \mathrm{M}$ website views per year

At BMC, research is always in progress.

Learn more biomedcentral.com/submissions 